\title{
Observation and simulation of space-charge effects in a radio-frequency photoinjector using a transverse multibeamlet distribution
}

\author{
M. Rihaoui, ${ }^{1,2}$ P. Piot, ${ }^{1,3}$ J. G. Power, ${ }^{2}$ Z. Yusof, ${ }^{2}$ and W. Gai ${ }^{2}$ \\ ${ }^{1}$ Northern Illinois Center for Accelerator \& Detector Development and Department of Physics, Northern Illinois University, \\ DeKalb, Illinois 60115, USA \\ ${ }^{2}$ High Energy Physics Division, Argonne National Laboratory, Argonne, Illinois 60439, USA \\ ${ }^{3}$ Accelerator Physics Center, Fermi National Accelerator Laboratory, Batavia, Illinois 60510, USA
}

(Received 25 March 2009; published 29 December 2009)

\begin{abstract}
We report on an experimental study of space-charge effects in a radio-frequency (rf) photoinjector. A $5 \mathrm{MeV}$ electron bunch, consisting of a number of beamlets separated transversely, was generated in an rf photocathode gun and propagated in the succeeding drift space. The collective interaction of these beamlets was studied for different experimental conditions. The experiment allowed the exploration of space-charge effects and its comparison with 3D particle-in-cell simulations. Our observations also suggest the possible use of a multibeam configuration to tailor the transverse distribution of an electron beam.
\end{abstract}

DOI: 10.1103/PhysRevSTAB.12.124201

PACS numbers: 29.27.- a, 41.85.- $\mathrm{p}$, 41.75.Fr

\section{INTRODUCTION}

Many accelerator applications call for the production of high charge (few $\mathrm{nC}$ ), low transverse emittance (few $\mu \mathrm{m}$ ), high peak current (several kA) electron bunches. Such applications include accelerator-based light sources [1], high energy physics accelerators [2], novel acceleration techniques, e.g., based on dielectric wakefield accelerators [3]. The dynamics of such generally space-chargedominated electron beams is intricate: it can develop transverse instabilities and is subject to phase space diluting effects [4,5]. Typically, high-brightness electron bunches are produced in a photoinjector: a high quantum efficiency photocathode, illuminated by a laser, is located on the back plate of a radio-frequency (rf) resonant cavity. High charge bunches are photoemitted and rapidly accelerated to relativistic energies [6] and the electron bunch properties are strongly affected by the photocathode drive laser parameters.

Several theoretical and experimental studies have previously addressed detrimental effects due to an inhomogeneous initial distribution in a rf photoinjector, e.g., photocathode drive laser transverse distribution nonuniformities. In most investigations, the beam is statistically characterized using its root-mean-square (rms) properties. For instance, Ref. [7] experimentally explores the impact of a transverse modulation on the rms transverse emittance. Although this is a universal characterization relying on the concept of "equivalent beam" [8,9] important details of the beam evolution might be missed. The pioneering work of Reiser and co-workers underlined the importance of studying the evolution of the beams transverse distribution in contrast to its rms properties only [10,11]. One of the experiments conducted at University of Maryland investigated the propagation of a $5 \mathrm{keV}$ DC beam, composed of five beamlets, in a "long" periodically focusing beam line.
The beamlets, arranged in a quincunx pattern, were generated by intercepting the beam with a mask downstream of a thermionic electron source. The experiment unveiled important physics related to phase mixing in chargedparticle beam and associated effects such as emittance dilution and halo formation. The latter experiment was recently repeated at the University of Maryland Electron Ring [12]. In both cases, the beamlets were created after the beam had reached $\sim 5-10 \mathrm{keV}$.

In this paper, we present the results of a similar experiment performed in an rf photoinjector using a $\sim 5 \mathrm{MeV}$ bunched electron beam. In contrast to the previous experiments, the electrons in our beam do not go through many betatron oscillations (they do not even make one oscillation) and the beamlets interact from their creation at the cathode. Masking the transverse distribution of the photocathode drive laser allowed the generation of easily controllable beamlets patterns. The evolution of the beamlets transverse density provides information on transverse space-charge effects which are validated against particlein-cell (PIC) simulations. Furthermore, our experimental observations point to a possible use of multibeamlet configuration to transversely control a beam. This could lead to new ways of manipulating an electron beam using space-charge interaction between several beams in a photoinjector. Several schemes based on space-charge interaction have been proposed as ways to control or shape charged-particle beams. For instance, Ref. [13] discusses the design of a very fast kicker based on such a scheme. Similarly, a technique using an electron lens to compensate tune shift induced by beam-beam effects in circular colliders was recently demonstrated in the Tevatron collider at Fermilab [14]. Finally, the generation of multiple-beamlet beams discussed in this paper has relevance to heavy ion fusion research [15] and intensity modulated radiation therapy [16]. 


\section{EXPERIMENTAL SETUP}

The experiment was performed at the Argonne Wakefield Accelerator (AWA) [17]; see Fig. 1. The accelerator incorporates a photoemission source, henceforth referred to as the rf gun, consisting of a $1+1 / 2$ cell $\mathrm{rf}$ cavity operating at $f=1.3 \mathrm{GHz}$ in the $\mathrm{TM}_{010, \pi}$ mode. An ultraviolet (uv) laser beam impinges a magnesium photocathode located on the back plate of the rf gun half cell. The thereby photoemitted electron bunch exits from the rf gun with a maximum kinetic energy of approximately $5 \mathrm{MeV}$ and is allowed to drift for a few meters with no external field save for the solenoid magnets. Two $100 \mu \mathrm{m}$ thick Ce:YAG screens [18] are used to measure the beam's transverse distribution. An integrated current monitor [19] provides the charge per bunch. The uv laser system consists of a titanium sapphire laser amplified with a regenerative amplifier and two linear amplifiers [20]. The infrared laser is frequency tripled to $\lambda=248 \mathrm{~nm}$ and is amplified in a single-stage krypton fluoride excimer amplifier. The laser system is located $\sim 20 \mathrm{~m}$ from the photocathode and the laser beam is transported via an optical 1:1 imaging system.

The rf gun is surrounded by three independently powered solenoidal magnetic lenses and referred to as L1, L2, and L3; see Fig. 1. During the experiment reported in this paper only L3 was used. The transverse distribution of the uv laser on the photocathode can be measured from a duplicate image of the laser beam on a "virtual photocathode." The virtual photocathode consists of a uv sensi-

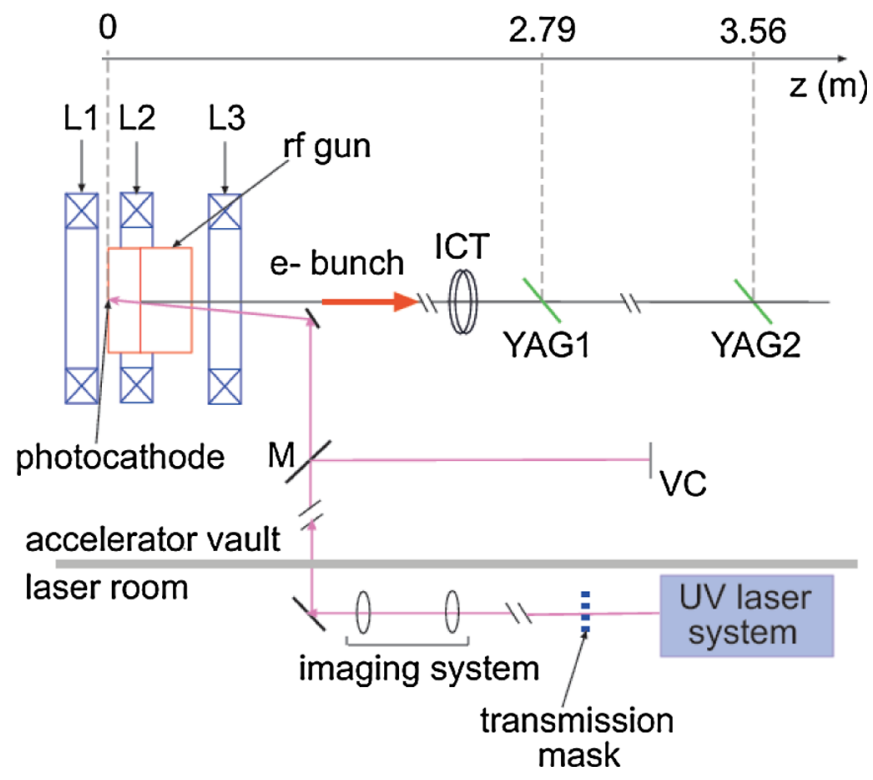

FIG. 1. (Color) Overview of the AWA beam line. Here, only the elements pertaining to our experiment are shown. The legend represents solenoidal magnetic lenses (L), optical mirror (M), virtual cathode (VC), integrated current monitor (ICT), and transverse profile monitor (YAG1 and 2). The distance along the beam line is also shown.
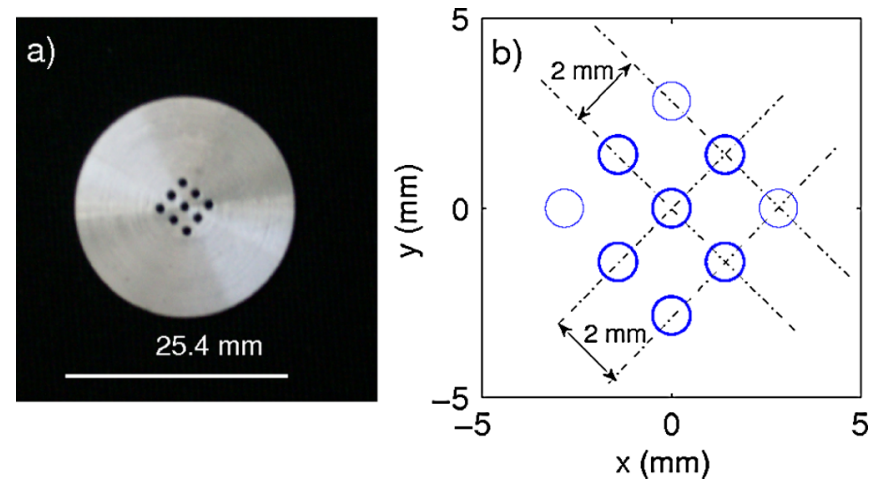

FIG. 2. (Color) Picture (a) of the aluminum mask used to shape the laser beam into a series of transversely separated beamlets and corresponding schematic of the mask geometry (b). In the experiments reported in this paper only five or six holes were not obstructed [shown as thicker line circles in (b)] and the mask was oriented to yield the pattern shown in (b) on the virtual photocathode [see also Fig. 4 (left image) for the corresponding uv laser transverse density].

tive CCD camera, located outside of the vacuum chamber, and is a one-to-one optical image of the laser on the photocathode.

The transverse distribution on the photocathode was controlled with a mask, located in the object plane of the imaging system, with a series of identical holes that could be selectively blocked (see Fig. 2). This object plane is located in the laser room thereby allowing laser shaping without interrupting the accelerator operation. The mask's center-to-center hole separation is $2 \mathrm{~mm}$ and the hole diameter is $1 \mathrm{~mm}$. The uv laser beam temporal profile was measured with a streak camera and can be approximated by a Gaussian distribution with rms duration of $\sigma_{t} \simeq 2 \mathrm{ps}$.

\section{EXPERIMENTAL RESULTS AND COMPUTER SIMULATIONS}

The evolution of the beamlet pattern was explored for two cases of total charge: $Q=20$ and $1000 \mathrm{pC}$, respectively referred to as "low" and "high" charge cases henceforth.

\section{A. Space-charge effects and solenoid focusing nonlinearities}

In this section we begin by estimating the role of space charge in the two regimes explored in the experiment. At a given axial location $z$ with respect to the photocathode, a measure of the importance of space-charge effects in driving the beam dynamics is given by the Debye length

$$
\lambda_{D}(z)=\frac{\varepsilon_{\perp}(z)}{2}\left[\gamma(z) \beta(z) \frac{I_{0}}{I(z)}\right]^{1 / 2} .
$$

This expression is obtained by using the properties of a beam bunch corresponding to its "rms equivalent uniform 
ellipsoid" [21]; $I(z) \equiv Q /\left[\sqrt{5} \sigma_{t}(z)\right], I_{0}=17 \mathrm{kA}$ denotes the Alfvèn current, $\varepsilon_{\perp}(z)$ is the transverse normalized rms emittance, $\gamma(z)$ is the beam's Lorentz factor, and $\beta(z) \equiv$ $\left[1-\gamma(z)^{-2}\right]^{1 / 2}$. Comparing the Debye length to the mean interparticle distance $\Lambda_{p}$ and the beam transverse size $\sigma_{\perp}$ provides an estimate of the importance of space-charge effects. If $\lambda_{D} \gg \sigma_{\perp}$ single-particle dynamics dominates whereas $\Lambda_{p} \ll \lambda_{D}<\sigma_{\perp}$ indicates space-charge effect are substantial. Finally, if $\lambda_{D} \sim \Lambda_{p}\left(<\sigma_{\perp}\right)$ particle-to-particle interactions become important. The quincunx beam distribution considered in this paper has two characteristic lengths: (1) the transverse rms size of the individual $\sigma_{\perp, i}$ $(i=1, \ldots, 5)$ and (2) the center-to-center separation between the beamlets $\delta(z)$ (at the cathode surface $\delta(z=0) \simeq$ $2 \mathrm{~mm}$ ). A quantity to assess the overall dominance of space-charge force over emittance effects is the averaged ratio $\bar{\varrho} \equiv 1 / L \int_{0}^{L} \sigma_{\perp}(z) / \lambda_{D}(z) d z$, where $L$ is the length of the accelerator beam line. A value of $\bar{\varrho} \gg 1$ indicates that space-charge forces are dominant over emittance effects in driving the beam dynamics. For typical values of the beam parameters considered in this paper, we find $\bar{\varrho} \leq 0.3$ for $Q=20 \mathrm{pC}$ and $\bar{\varrho} \geq 1.7$ for $Q=1 \mathrm{nC}$. These simple estimates indicate that in general space charge plays a significant role in the beam dynamics of our experiment.

On the other hand, comparing the Debye length to the distance between the beamlets indicates that the force between the beamlet gives $\delta(z \simeq 0) / \lambda_{D}>1$ for both the high and low charge cases. This indicates that the interbeamlet dynamics is going to be governed by the typical (point-to-point) Coulomb force $\propto Q^{2} / \delta^{2}(z \simeq 0)$ (in contrast to a smoothed potential). Hence near the photocathode, where space-charge effects are the most prominent, the interbeamlet forces in the high charge case are going to be $\sim(1000 / 20)^{2}=2500$ times larger than in the low charge regime. Note that this value is overestimated since we assumed the five beamlets to behave as pointlike macroparticles with charge $Q / 5$. In addition, the situation is complicated by the fact that the beamlets overlap as they propagate along the accelerator beam line (but this happens for $z>1 \mathrm{~cm}$ ). Nevertheless our estimates show that spacecharge effects are going to be far more important in governing the interbeamlet beam dynamics for the high charge case compared to the low charge case.

In addition, the beam considered in this paper is composed of multiple beamlets and could potentially have a large (several $\mathrm{mm}$ ) transverse beam size. It is therefore important to assess the nonlinearities in the transverse focusing force introduced by the solenoid. These nonlinearities are estimated by Taylor expanding the radial component of the magnetic field to next (third) order in radial position $r$ with respect to the solenoid axis

$$
B_{r}(r, z)=-\frac{r \hat{B}}{2} \frac{\partial B}{\partial z}+\frac{r^{3} \hat{B}}{16} \frac{\partial^{3} B}{\partial z^{3}}+\mathcal{O}\left(r^{5}\right),
$$

where $B \equiv B_{z}(r=0, z)$ is the normalized axial magnetic
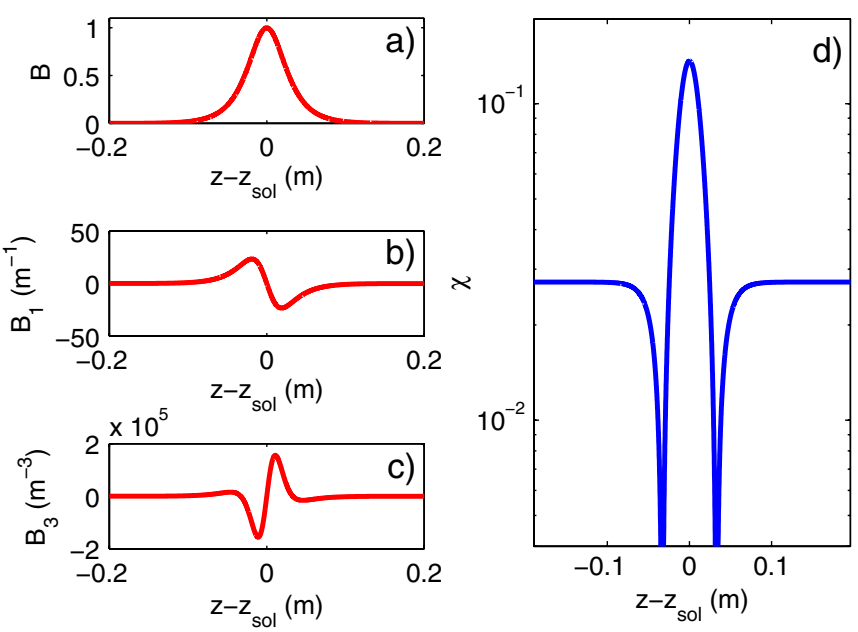

FIG. 3. (Color) Axial normalized magnetic field of L3 (a), first $B_{1} \equiv \frac{\partial B}{\partial z}(\mathrm{~b})$, and third $B_{3} \equiv \frac{\partial^{3} B}{\partial z^{3}}$ (c) derivatives and nonlinearover-linear focusing ratio $\chi$ computed at $r=1 \mathrm{~cm}$ (d). In plot (a) both the magnetic field obtained from POISSON simulation (blue) and its analytical approximation (red) are shown. The analytical function is $\tilde{B}(z)=1 / \cosh (z / 0.0214)$. The horizontal axes of all the plots have their origins coincident with the solenoid's center located at $z_{\mathrm{sol}} \simeq 0.57 \mathrm{~m}$ from the photocathode.

field profile and $\hat{B}$ is the peak value of the magnetic field. The ratio of the nonlinear-over-linear terms is given by

$$
\chi \equiv\left|\frac{8}{r^{2}} \frac{\partial B}{\partial z}\left(\frac{\partial^{3} B}{\partial z^{3}}\right)^{-1}\right|
$$

The magnetic field $B$ was computed with POISSON [22] and approximated with the function $\tilde{B}(z)=a / \cosh (z / b)$ [23]; see Fig. 3, plot (a). The analytical function was used to computed the needed derivatives [see Fig. 3, plots (b) and (c)] to eventually obtain an analytical expression for the ratio $\chi$. Figure 3, plot (d), shows the evolution of $\chi$ inside the solenoid at a radius of $r=1 \mathrm{~cm}$. A maximum value of $\chi \simeq 0.1$ is reached at the center of the solenoid. In the simulations presented in this paper the particles, while propagating through the solenoid, are always contained within a maximum radius of $\sim 7 \mathrm{~mm}$ so the nonlinear force experienced by a particle at such a radius is, at most, only $\sim 5 \%$ of the linear focusing force. Therefore the solenoid nonlinearity does not contribute significantly to the results reported herein.

\section{B. Low charge regime}

The operating conditions of the main subsystems of the photoinjector used during the experiment reported below are gathered in Table I. A first experiment consisted in generating a low charge electron beam $(Q=20 \mathrm{pC})$ comprising six beamlets arranged in an asymmetric pattern; see Fig. 4. This asymmetric beamlet configuration allows us to identify the orientation of the pattern on both the virtual cathode and YAG1. Because of weak space-charge effects, 
TABLE I. Nominal accelerator settings during the experiment. The "Experiment" and "Model" columns respectively correspond to values inferred from the AWA control system and values obtained when matching the impact- $T$ model to the single-particle measurements.

\begin{tabular}{lcc}
\hline \hline Parameter (unit) & Experiment & Model \\
\hline Laser rms pulse duration (ps) & $1.9 \pm 0.2$ & 1.9 \\
Laser injection phase (deg) & $50 \pm 5$ & 53 \\
E-field on cathode $(\mathrm{MV} / \mathrm{m})$ & $54 \pm 3$ & 51 \\
L3 peak axial B-field (T) & {$[0.30,0.40] \pm 0.01$} & {$[0.30,0.40]$} \\
Charge-high charge (nC) & $0.9 \pm 0.1$ & 0.9 \\
Charge-low charge (nC) & $0.015 \pm 0.005$ & 0.015 \\
Kinetic energy (MeV) & $5.20 \pm 0.10$ & 5.28 \\
\hline \hline
\end{tabular}

the beamlets do not interact strongly and effectively behave as independent macroparticles. The observed pattern rotation between the photocathode and YAG1, visible in Fig. 4, is due to the Larmor precession induced as the beam propagates through the L3 magnetic lens. The rotation angle is given by [24]

$$
\theta(z)=\hat{B} \int_{0}^{z}\left[e B\left(z^{\prime}\right)\right] /\left[2 m \gamma\left(z^{\prime}\right) \beta\left(z^{\prime}\right) c\right] d z^{\prime}
$$

where $e$ is the electronic charge and $m$ the rest electron mass. The rotation angle downstream of the solenoid field, $\theta(\infty)$, was calculated by numerically integrating Eq. (4) using the axial magnetic field obtained from POISSON while $\beta(z)$ was computed with IMPACT-T [25]. The calculated rotation angle downstream of the solenoidal field was found to be $\theta(\infty)=-32 \pm 2 \mathrm{deg}$ (the quoted uncertainty comes from the uncertainty on the peak E-field in the rf gun). The computed value compares well with the measured rotation angle of $\theta(\infty)=-34.7 \pm 2.6 \mathrm{deg}$ for a peak axial magnetic field $\hat{B}=0.339 \mathrm{~T}$.

To aid in understanding the experiment, we performed 3D PIC simulations of the beam dynamics using the program IMPACT-T. The code uses a quasielectrostatic space-
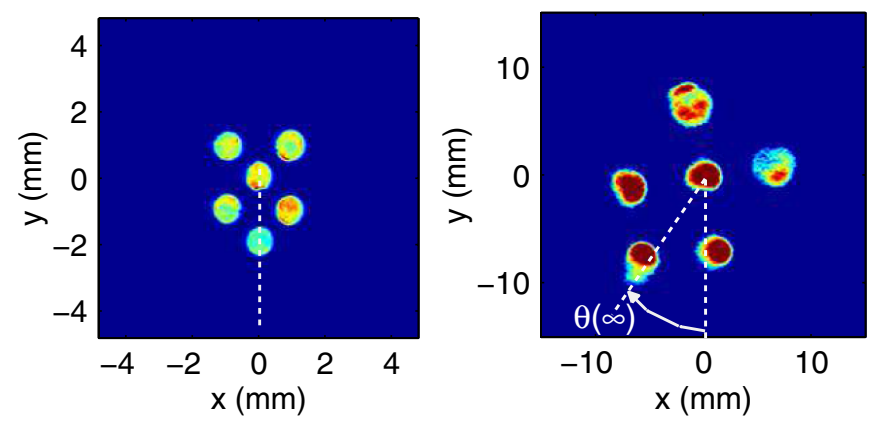

FIG. 4. (Color) False color image of the measured uv laser transverse distribution on the virtual cathode (VC) location (left) and corresponding measured electron beam distribution at YAG1 location (right). The total charge is $Q=20 \pm 2 \mathrm{pC}$. charge algorithm where the force in the laboratory frame is found by solving Poisson's equation in the bunch's rest frame [25]. IMPACT-T was run with a simplex optimizer [26] to fine-tune the operating parameters of the accelerator used in the model to match the low charge measurements. During the optimization process the E-field on the cathode, the laser launch phase, and L3 peak axial B-field were varied to ensure that the simulation of the low charge quincunx pattern reproduced the low charge measurement on YAG1 (shown in Fig. 4). The minimization criterion for the optimization was to match the measured and simulated positions of the beamlets' centroid. The five beamlets were thereby treated as independent transversely separated macroparticles and matching the evolution of their centroids provides a validation of our model in the single-particle dynamics regime. The set of parameters obtained are compiled in Table I and are in decent agreement with the settings inferred from the accelerator control system.

\section{High charge regime}

As the total charge associated with the multibeamlet distribution is increased to $\sim 1 \mathrm{nC}$, space-charge effects become significant which results in a change in: (1) the intradynamics within the beamlets and (2) the interactions between the beamlets. Such interactions resulted in the development of unexpected features that depend on the initial pattern [27].

At high charge, 3D simulations including the measured transverse laser density were performed. The initial 3D spatial distribution of macroparticles used in our numerical simulations was generated with a quasirandom generator using the digitized pictures of the laser transverse density captured on the virtual cathode. The algorithm, presented in the Appendix, can also be used to generate a temporal distribution based on a measured profile (from a streak camera, for example). However, in this experiment the laser was taken to have a Gaussian temporal distribution with a rms duration of 2 ps consistent with streak camera measurements. The corresponding initial momentum distribution of the macroparticles was generated to follow an isotropic distribution in the forward half sphere. The momentum modulus of each macroparticle is computed from the excess kinetic energy taken to be $1 \mathrm{eV}$ in the present case.

In Fig. 5, we present the measured and simulated transverse distributions at YAG1 for different values of the L3 magnetic fields $\hat{B}$. The simulations qualitatively reproduce the main features observed experimentally, especially the formation of radial tails on the periphery of the beamlets. For large values of the magnetic field the central beamlet appears confined. This focusing effect resulting from the presence of the peripheral beamlets is confirmed by masking the surrounding beamlets: the central beamlet rms transverse beam size increases from $1.1 \pm 0.3 \mathrm{~mm}$ to $3.9 \pm 0.3 \mathrm{~mm}$; see Fig. 6 . 

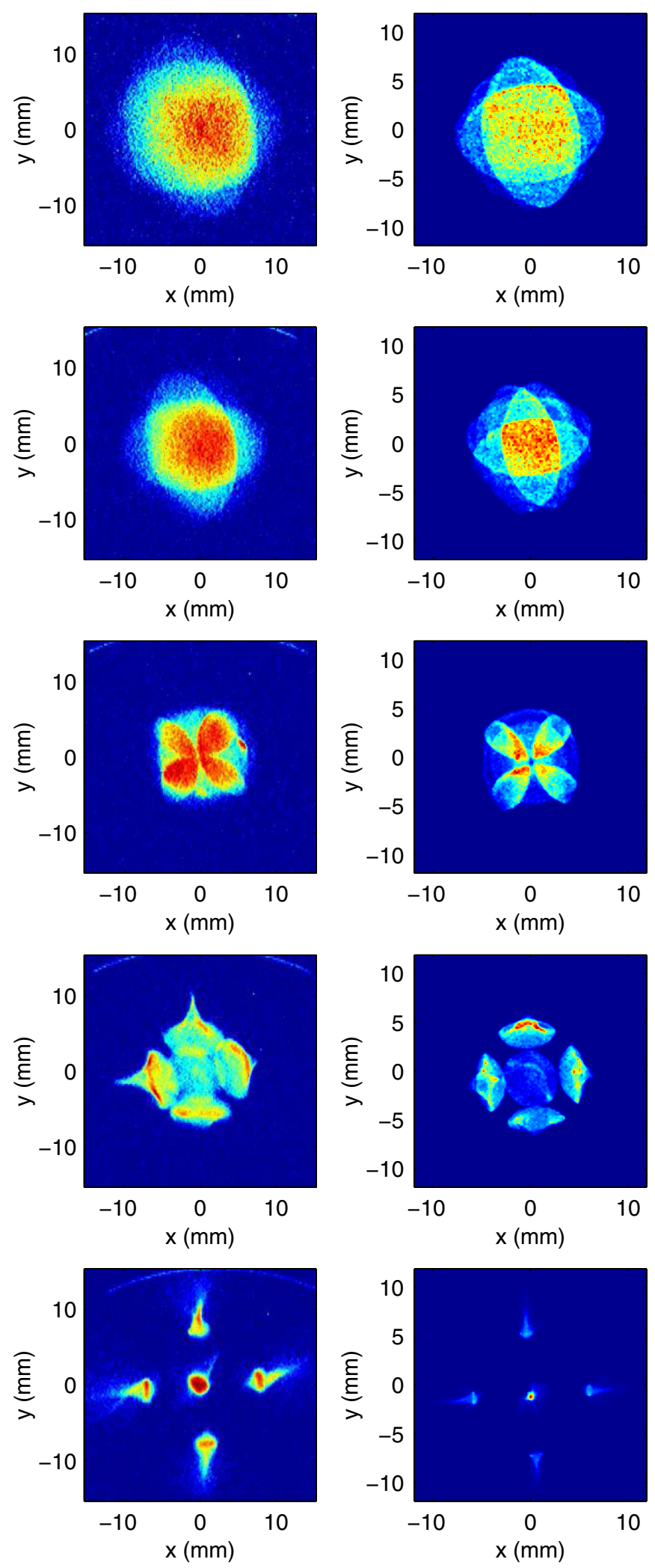

FIG. 5. (Color) False color images of the measured (left column) and simulated (right column) electron beam transverse density distribution at YAG1 location. The five rows respectively correspond to the different values of the magnetic field (from top row) $\hat{B}=0.333,0.339,0.350,0.359$, and $0.376 \mathrm{~T}$.
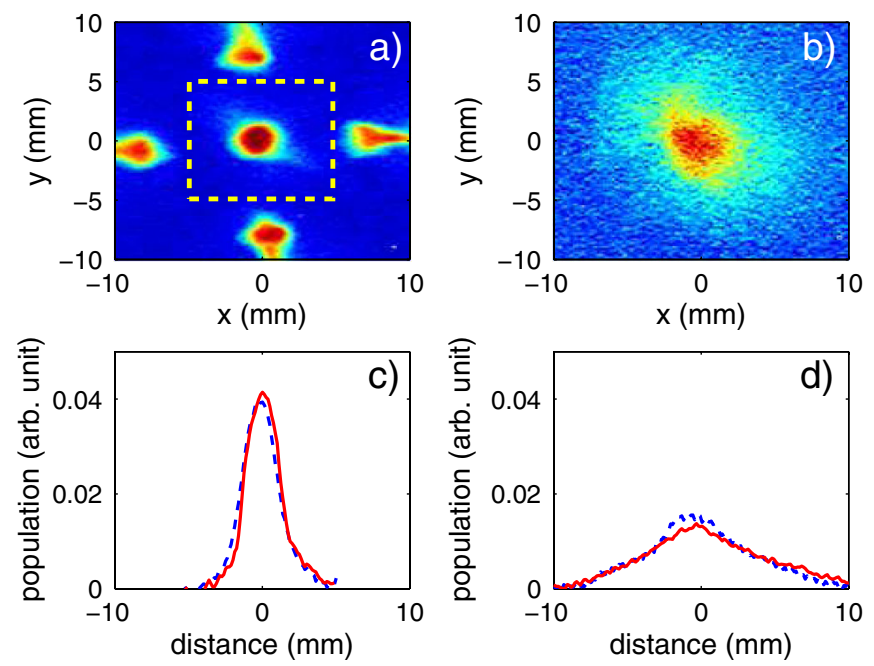

FIG. 6. (Color) Measured two-dimensional transverse beam density at YAG1 location and corresponding horizontal (blue dashed line) and vertical (red solid line) projections for the cases with $[Q=0.93 \pm 0.05 \mathrm{nC}][(\mathrm{a}),(\mathrm{c})]$ and without $[(\mathrm{b}),(\mathrm{d})][Q=$ $0.17 \pm 0.03 \mathrm{nC}]$ surrounding beamlets. The dashed box in (a) represents the region of interest used to compute the projections displayed in (c). The magnetic field is $\hat{B}=0.376 \mathrm{~T}$.

\section{ANALYSIS}

Given the qualitative agreement between simulations and measurements shown in the preceding sections, it remains to understand the origin of the observed features. The primary low charge effect, pattern rotation, has already been shown to be due to the Larmor rotation of the beam upon passing through the solenoidal field. At high charge, there are two notable features that we wish to understand: the change in the focusing of the central beamlet and the formation of radial tails on the periphery of the outer beamlets. In this section, we use numerical simulations to provide insight into the physical mechanism responsible for these observed features.

The initial conditions considered in the present section are similar to the experimental conditions. The only difference is the laser distribution which is now "idealized" by considering the individual beamlets to have a uniform transverse density (instead of using the measured laser distribution). The introduction of such an idealized laser distribution is motivated by our desire to understand the main features associated with our observations, but without adding complexity to the observed laser nonuniformities. During the experiment, we tried to produce radially uniform beamlets and deviations from this distribution are small and fluctuate from shot to shot.

\section{A. Larmor Coordinate System}

To simplify the analysis of the simulation results we introduce a rotating coordinate system $\Xi \equiv\left(\xi, p_{\xi}, \zeta, p_{\zeta}\right)$ which is related to the laboratory coordinate system by 


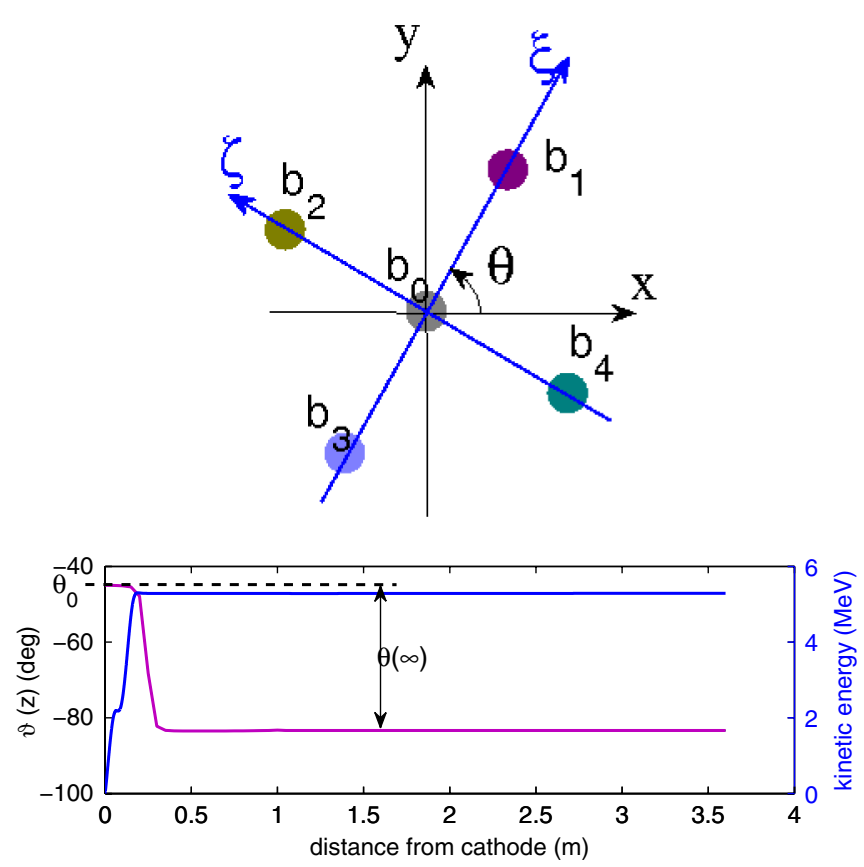

FIG. 7. (Color) Definition of the Larmor $(\xi, \zeta)$ and laboratory $(x, y)$ transverse coordinate systems (top schematics). Evolution of the rotation angle $\vartheta(z) \equiv \theta_{0}+\theta(z)$ between the $(x, y)$ and Larmor $(\xi, \zeta)$ frames (magenta trace) and of the beam's kinetic energy (blue trace) along the accelerator beam line for $\hat{B}=$ $0.376 \mathrm{~T}$ (bottom plot). In this example $\theta_{0}=-45^{\circ}$ and $\theta(\infty) \simeq$ $-38^{\circ}$.

$\Xi(z)=R\left[\theta_{0}+\theta(z)\right] \mathbf{X} \quad$ where $\mathbf{X} \equiv\left(x, p_{x}, y, p_{y}\right) . \quad$ The quantities $\theta_{0}$ and $\theta(z)$ are the initial rotation angle of the quincunx pattern on the photocathode and the Larmor rotation angle, respectively. $R$ is a four-dimensional rotation matrix,

$$
R[\vartheta(z)]=\left[\begin{array}{cc}
I \cos \vartheta(z) & I \sin \vartheta(z) \\
-I \sin \vartheta(z) & I \cos \vartheta(z)
\end{array}\right],
$$

wherein $I$ is the $2 \times 2$ identity matrix and $\vartheta(z) \equiv \theta(z)+$ $\theta_{0}$. The angle $\theta_{0}$ is chosen such that $\xi$ and $\zeta$ axes correspond to the two symmetry axes of the quincunx pattern and $\theta(z)$ is numerically computed using Eq. (4); see Fig. 7. The initial pattern rotation angle on the photocathode is $\theta_{0}=-45^{\circ}$ and $\theta(z=0)=0$. Working in the Larmor coordinate system avoids the complications of dealing with coupling effects between the two transverse degrees of freedom. The trajectories and rms transverse envelopes of the five beamlets in the Larmor frame are presented in Fig. 8 for $\hat{B}=0.376 \mathrm{~T}$. The beamlets' trajectories intersect at $z \simeq 1.3 \mathrm{~m}$ and the envelopes strongly overlap for $z \leq$ $1 \mathrm{~m}$.

\section{B. Beam dynamics associated to the central beamlet}

As discussed in the previous section, the presence of the peripheral beamlets affects the dynamics of the central beamlet at YAG1's location. Once again, this feature is
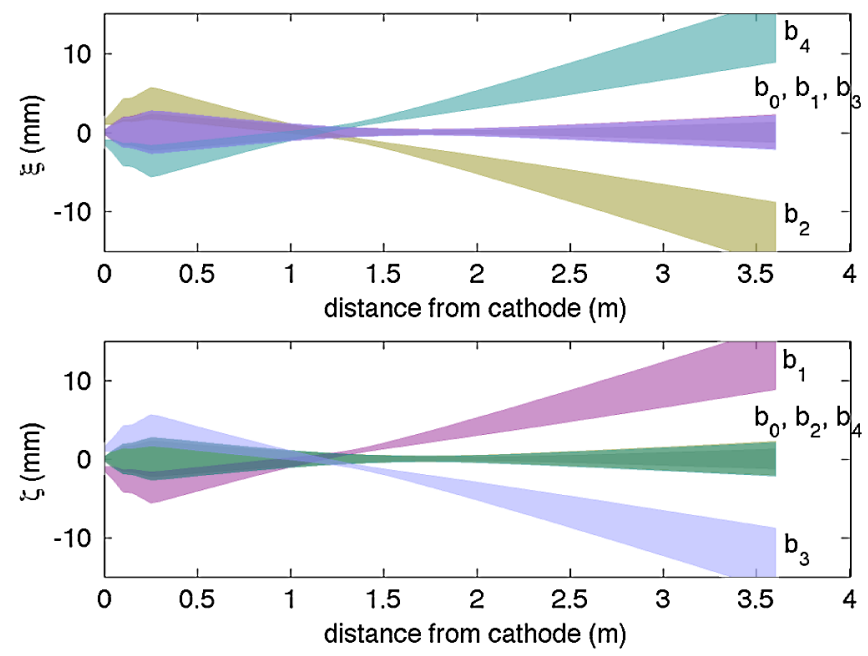

FIG. 8. (Color) Beamlets' transverse rms envelopes and trajectories in the $(z, \xi)$ (top) and $(z, \zeta)$ (bottom) for $\hat{B}=0.376 \mathrm{~T}$. The $\mathrm{b}_{i}$ labels correspond to the beamlets shown in Fig. 7 (top).

investigated and reproduced via numerical simulations. IMPACT-T calculations performed for various solenoid settings indicate that the surrounding beamlets affect the central beamlet transverse size and normalized emittance

$$
\varepsilon_{\perp} \equiv\left[\left\langle\left(\beta_{\perp} \gamma\right)^{2}\right\rangle\left\langle x_{\perp}^{2}\right\rangle-\left\langle x_{\perp}\left(\beta_{\perp} \gamma\right)\right\rangle^{2}\right]^{1 / 2},
$$

where $x_{\perp}$ is the considered transverse coordinate, $\beta_{\perp} \equiv$ $v_{\perp} / c$ (with $v_{\perp}$ being the component of the velocity along $\hat{x}_{\perp}$ ). The angular brackets in the latter equation stand for the statistical averaging over the phase space distribution.

The evolution of the central beamlet rms size and emittance is illustrated in Fig. 9 for various cases of solenoid settings and with (or without) the peripheral beamlets. The evolution and optimization of the transverse emittance of space-charge-dominated beams produced in photoinjectors has been the subject of extensive investigations [28-30]. It was especially shown that there is an optimum condition, corresponding to a particular value of $\hat{B}$, that can minimize the final emittance at a given location. Therefore varying the value of $\hat{B}$ is expected to impact the evolution of the transverse emittance as illustrated in Fig. 9 (red dashed lines) for the case of single beamlet (the central one). The presence of the peripheral beamlets influences the focusing of the central beamlet and therefore impact the emittance evolution as supported by comparing the green and blue traces shown in Fig. 9.

Among the various configurations explored, the one where the central beamlet is surrounded by the peripheral beamlets with $B=0.376 \mathrm{~T}$ is the only one capable of maintaining a small beam size while preserving its transverse emittance nearly constant downstream of the rf gun and solenoid (i.e. for $z>0.5 \mathrm{~m}$ ). The resulting focusing at the location of YAG1 is due to the beamlet interactions upstream of the solenoid. In this region $(z<0.2 \mathrm{~m}$ in Fig. 9) the presence of the outer beamlets reduces the 

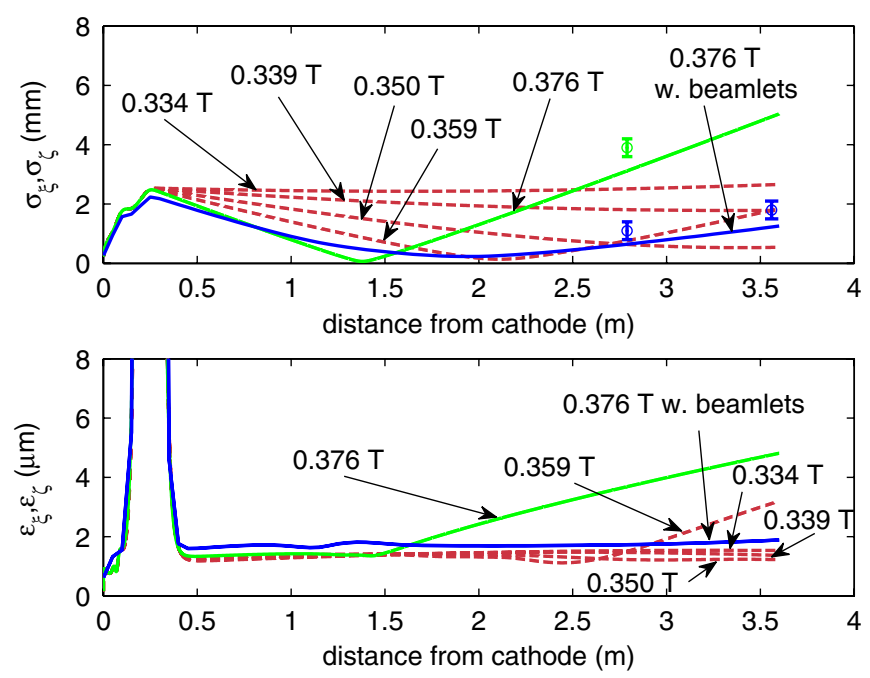

FIG. 9. (Color) Simulations of transverse rms beam size (top) and emittance (bottom) of the central beamlet along the $z$ axis for different values of L3 peak axial magnetic field $\hat{B} \in$ $[0.339,0.376] \mathrm{T}$ (dashed red lines). The cases shown in Fig. 6 correspond to the blue (with surrounding beamlets) and green (without surrounding beamlets) solid lines. The corresponding data measured at YAG1 and YAG2 are also shown as dots with the same color coding convention as the simulations.

divergence of the central beamlet which results in a reduced focusing kick imparted by the solenoid. In the case of $\hat{B}=0.376 \mathrm{~T}$, the central beamlet alone is nominally focused $\sim 1.5 \mathrm{~m}$ upstream of the YAG1 (green trace in Fig. 9). The presence of the peripheral beamlets effectively moves the waist toward larger values of $z$ (blue trace in Fig. 9).

\section{Nonlaminar flow and radial mixing}

Further insight into the underlying beam dynamics can be inferred from color coding the macroparticles according to their initial radial coordinates when photoemitted. Such an analysis is presented in Figs. 10-12 for, respectively, the cases of the central beamlet alone, the central beamlet surrounded by the four peripheral beamlets, and for one of the peripheral beamlets.

The evolution of the transverse configuration space in the Larmor frame $(\xi, \zeta)$ (shown in the top row of Fig. 10) conforms to the typical evolution of a space-chargedominated beam in a solenoid and drift space: the radial hierarchy is preserved (laminar flow) as the beam goes through a waist (at $z \simeq 140 \mathrm{~cm}$ ) \{a closer examination shows a slight mixing due to (i) the nonuniform (Gaussian) longitudinal distribution of the bunch and (ii) chromatic effects originating from the quadratic dependency of the axial space-charge electric field on the radial coordinate [31,32]\}. By contrast, the top row of Fig. 11 presents atypical dynamics: as the beam goes through the waist (at $z \simeq 2.0 \mathrm{~m}$ ) the radial hierarchy is lost and the macroparticles redistribute in the phase space; a large fraction of the beam's population crosses over the beamlets central axis. Eventually, the macroparticles retain some degree of organization but the initial radial hierarchy is washed out (nonlaminar flow). The beam's transverse distribution is not cylindrically symmetric but acquires a square shape. The effect of the surrounding beamlets, inferred by comparing the transverse phase spaces plots shown in the bottom rows of Figs. 10 and 11, shows that their presence reduces the beam's initial divergence by $40 \%$. This reduced beam divergence and consequently reduced beam size results in a smaller focusing kick imparted by the solenoid.

Finally, the configuration space associated with one of the peripheral beamlets is shown in Fig. 12. In general, similar remarks as those just mentioned still hold, the main difference being the highly asymmetric distribution. Close inspection of the figure reveals that particles that begin in the outer radial layer of the noncentral beamlet near the
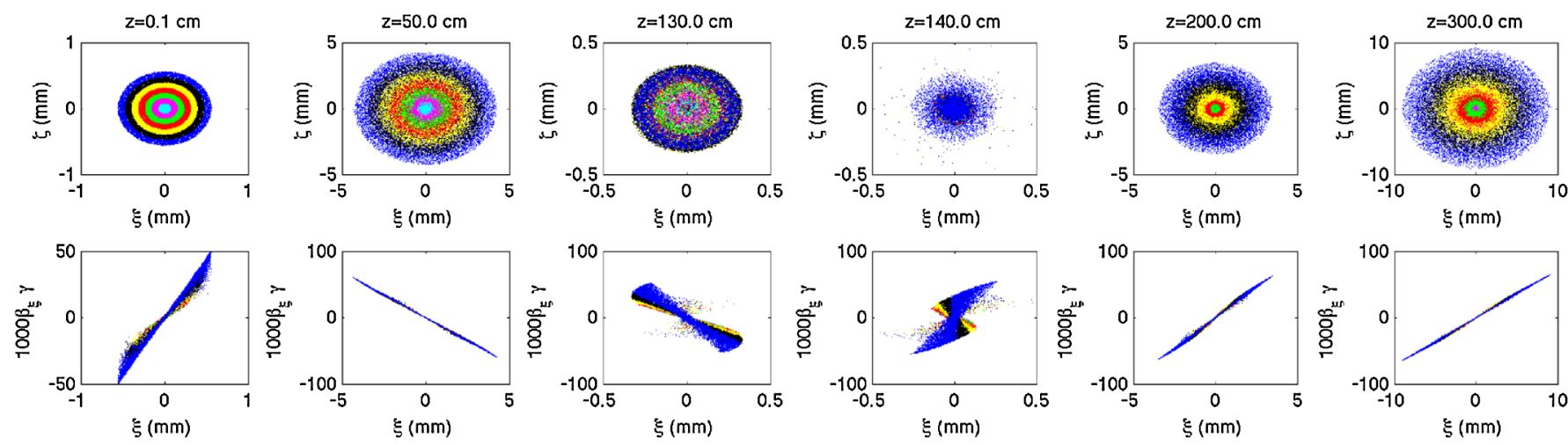

FIG. 10. (Color) Macroparticle distributions in the transverse configuration space (top) and transverse horizontal phase space (bottom) at different locations from the photocathode surface $(z=0.1,50$, and 130, 140, 200, and $300 \mathrm{~cm}$ from left to right columns) associated to the central beamlet without the peripheral beamlets. The peak B-field of L 3 is $\hat{B}=0.376 \mathrm{~T}$. The coordinate system is the Larmor frame $(\xi, \zeta)$. The color coding corresponds to different radial slices at the emission time (the beam at emission was divided into seven radial slices of same thickness $\delta r \simeq 70 \mu \mathrm{m}$ ). 

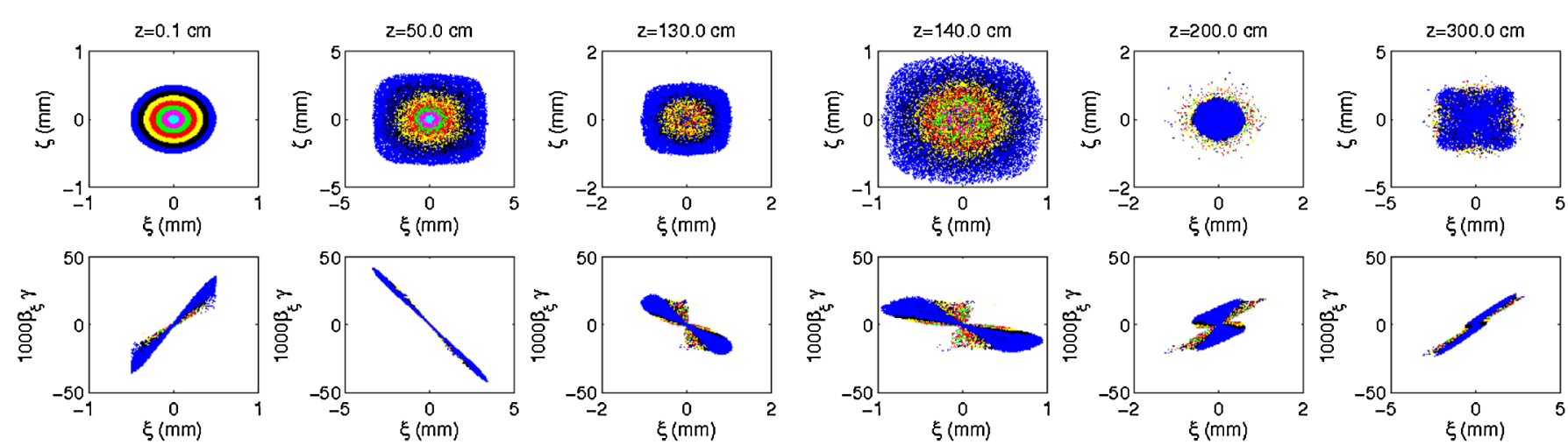

FIG. 11. (Color) Macroparticle distributions in the transverse configuration space (top) and transverse horizontal phase space (bottom) at different locations from the photocathode surface $(z=0.1,50$, and 130, 140,200, and $300 \mathrm{~cm}$ from left to right columns) associated to the central beamlet with the peripheral beamlets. The peak B-field of L3 is $\hat{B}=0.376 \mathrm{~T}$. The coordinate system is the Larmor frame $(\xi, \zeta)$. The color coding corresponds to different radial slices at the emission time (the beam at emission was divided into seven radial slices of same thickness $\delta r \simeq 70 \mu \mathrm{m}$ ).

photocathode are pushed into the radial tail (blue macroparticles) by space charge, as will be shown in the next section.

\section{Transverse beam dynamics in the rf gun}

Further insight into the mechanism that causes the tail formation can be gained by computing the transverse force associated with the density distribution as it propagates along the beam line. We perform such a calculation by numerically solving Poisson's equation in the bunch average rest frame. The thereby obtained scalar potential $\Phi$, tabulated on a three-dimensional grid, can directly be used to derive the transverse force a macroparticle would experience as it moves in the laboratory frame [33,34]. For our purposes, it is more convenient to compute the chargenormalized transverse component of the Lorentz force (in the laboratory frame) a macroparticle with coordinates $(\xi, \zeta, z)$ experiences:

$$
\mathbf{F}_{\perp}(\xi, \zeta, z) \simeq \frac{1}{\gamma^{2}}\left[\nabla_{\perp} \Phi\right](\xi, \zeta, z)
$$

where $\nabla_{\perp} \equiv \hat{\xi} \partial_{\xi}+\hat{\zeta} \partial_{\zeta}$ is the transverse gradient. The calculation of $\mathbf{F}_{\perp}$ was performed numerically in the Larmor frame $(\xi, \zeta, z)$. An example of the computed force integrated over the longitudinal bunch distribution, $\mathbf{F}_{\perp}(\xi, \zeta)$, is shown in Fig. 13 (bottom row) along with the corresponding $z$-integrated transverse density distributions (top row) for different locations along the accelerator beam line. As can be seen from the force plots, the beamlets begin interacting from their birth (see $z=0.1 \mathrm{~cm}$, Fig. 13), even before overlapping. As a result, the transverse force develops a twofold transverse symmetry. Using the techniques developed in this section we are now in a position to understand what causes the formation of the radial tails. Before applying this technique, it was assumed that the tail formation was due to the space-charge inter-
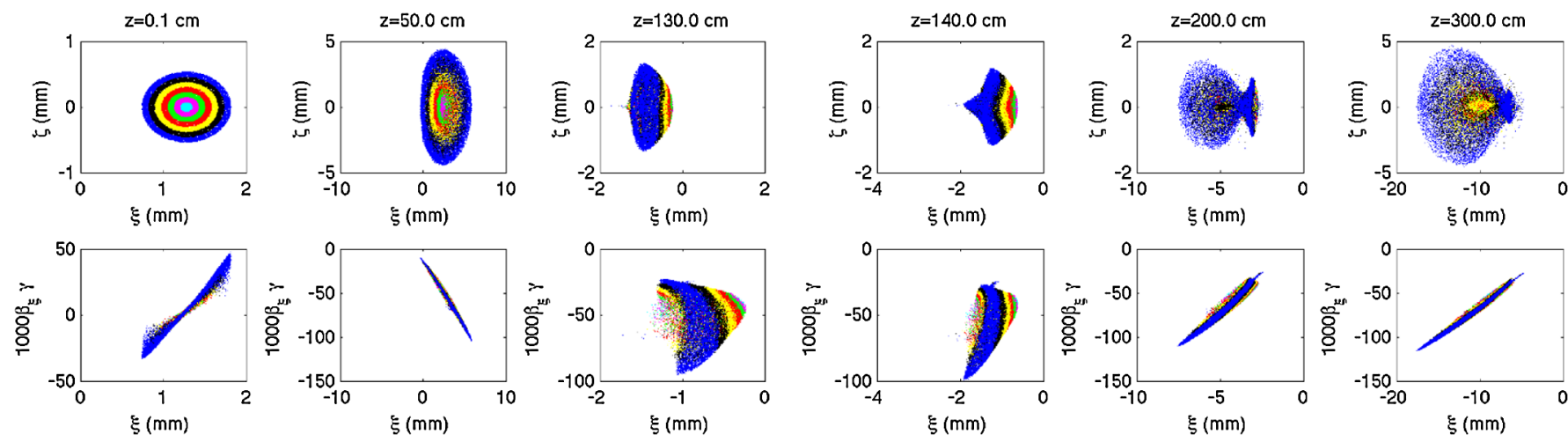

FIG. 12. (Color) Macroparticle distributions in the transverse configuration space (top) and transverse horizontal phase space (bottom) at different locations from the photocathode surface $(z=0.1,50$, and 130, 140,200, and $300 \mathrm{~cm}$ from left to right columns) associated to the peripheral beamlet $b_{1}$ in Fig. 7 (bottom row). The peak B-field of L3 is $\hat{B}=0.376 \mathrm{~T}$. The coordinate system is the Larmor frame $(\xi, \zeta)$. The color coding corresponds to different radial slices at the emission time (the beam at emission was divided into seven radial slices of same thickness $\delta r \simeq 70 \mu \mathrm{m}$ ). 

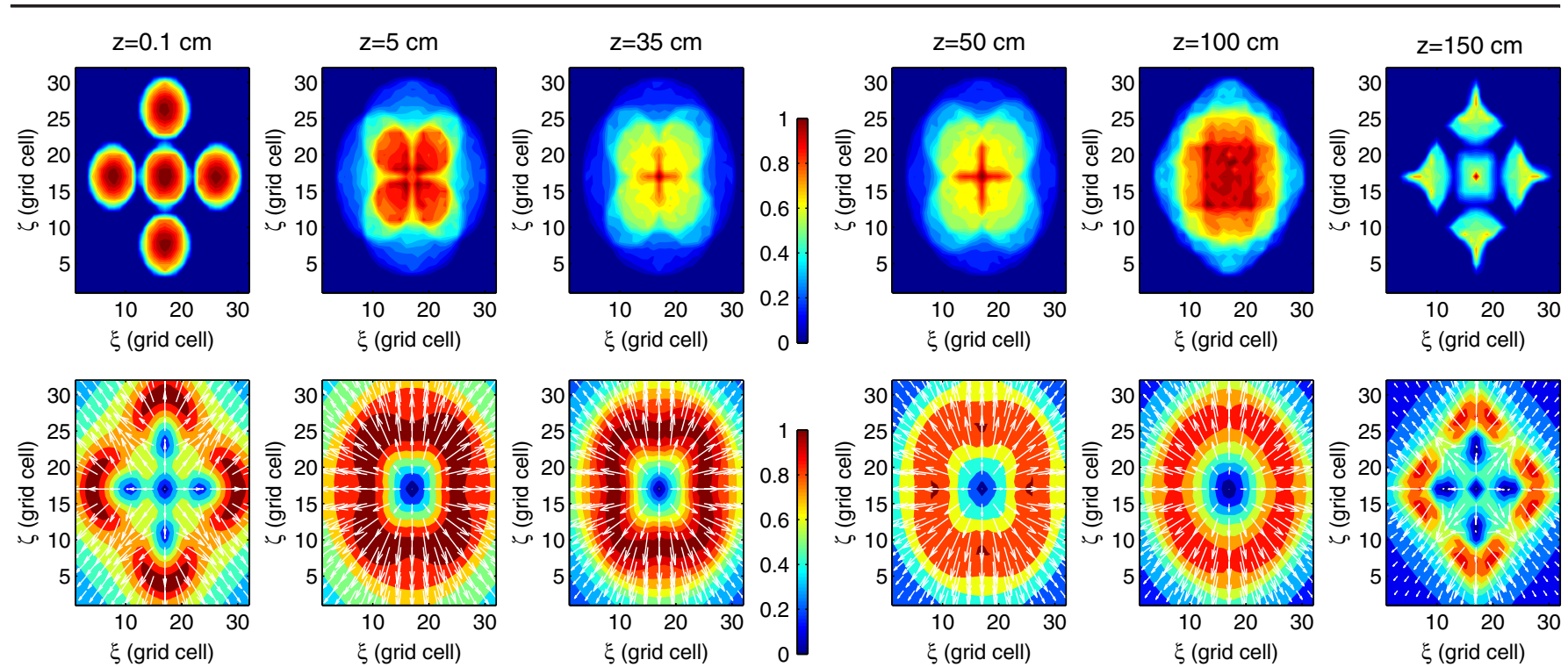

FIG. 13. (Color) $z$-integrated transverse distribution (top row) and corresponding charge-normalized transverse Lorentz force $\mathbf{F}_{\perp}$ [given by Eq. (3)] tabulated on a $32 \times 32$ transverse grid (bottom rows). The color intensity represents the number of macroparticle (top row) and the force modulus (bottom row). The arrows in the bottom row give the Lorentz force directions (the arrow's length is proportional to the force modulus). Each column corresponds to different position for the bunch center from the photocathode surface (as indicated on the top row) of $z=0.1,5,35,50,100$, and $150 \mathrm{~cm}$. The peak B-field of L3 is $\hat{B}=0.376 \mathrm{~T}$. The color coding convention for the density (respectively transverse force) is such that blue and red respectively correspond to lowest and largest electron population (respectively force modulus). Note that the plots are in units of the space-charge grid cells.

action at the beamlet crossover; approximately $z=$ $130 \mathrm{~cm}$. However, the effect indeed originates from the space-charge interaction at the early stage of the bunch generation and transport. In Fig. 13, it is seen that the space-charge force produces a stronger defocusing effect for the population in the outer region of the peripheral beamlets than for the ones in the inner region. While in the rf gun, the beamlets overlap (see Fig. 8 for $z<0.3 \mathrm{~m}$ ) and the resulting potentials have local minima which give rise to weaker defocusing forces along four directions (coincident with the two axes of symmetry); see Fig. 13 $(z=5,35$, and $50 \mathrm{~cm})$. Eventually, the beamlets crossover (e.g. $z=130 \mathrm{~cm})$ and separate $(z=150 \mathrm{~cm})$. Note that the space-charge force is nearly axially symmetric at the crossover and therefore incapable of imparting the fourfold symmetry seen in the overall pattern at $z=150 \mathrm{~cm}$. The shape of central beamlet becomes square due to the twofold symmetry of the transverse force while the peripheral beamlets have a onefold symmetry.

\section{SUMMARY}

In summary, we have observed and explained several space-charge induced features associated with the generation and propagation of a multibeamlet electron bunch in a rf photoinjector. We showed, via numerical simulations, that in the quincunx configuration the peripheral beamlets could provide a means to control the transverse size and shape of the central beamlet. It might be possible to design a photoinjector exploiting such a system. For instance, in electron sources based on superconducting rf guns, the use of an external magnetic field to focus the beam at an early stage can be difficult, and using a multibeamlet configuration could allow the solenoid to be located further downstream of the cathode. Similarly, the possibility to alter a beam using a set of photoemitted peripheral beamlets with a temporal profile different from the main beam could open new ways for spatiotemporal shaping of electron beams. In addition, such dynamic control of the transverse beam parameters could provide an alternative scheme for emittance compensation in space-charge-dominated electron beams [35].

\section{ACKNOWLEDGMENTS}

We are grateful to Dr. Ji Qiang from Lawrence Berkeley National Laboratory for providing us with a copy of IMPACT-T. The work of M.R. and P.P. was supported by the U.S. Department of Energy under Contracts No. DEFG02-04ER41323 and No. DE-FG02-08ER41532 with Northern Illinois University. W. G., J.P., and Z. Y. are supported by the U.S. Department of Energy under Contract No. DE-AC02-06CH11357 with Argonne National Laboratory.

\section{APPENDIX A: MEASUREMENT-BASED GENERATIONS OF 3D MACROPARTICLE DISTRIBUTIONS}

A Monte Carlo generator was developed to generate an initial 3D macroparticle distribution of $N$ macroparticles, 
$\left\{x_{k}, y_{k}, t_{k}\right\}$ where $k \in[1, N]$, at the photocathode based on the measured laser distribution. The laser distribution was assumed to be factorizable into separate transverse $I(x, y)$ and temporal $\mathcal{S}(t)$ distributions. The laser transverse distribution can be measured by digitizing the laser spot image on the virtual cathode; see Fig. 1. Given a typical eight-bit CCD camera, with a $640 \times 480$ pixel array, the measurement results in a matrix of pixel values $I_{i, j}$, where $i=[1,2, \ldots, 640], \quad j=[1,2, \ldots, 480]$, and $0 \leq I_{i, j} \leq$ 255. The measured temporal distribution (measured with a streak camera in our case) results in a vector $S_{q}$ with $q$ running over the number of pixels used in the measurement.

The generation of the transverse macroparticle distribution, $\left\{x_{k}, y_{k}\right\}$ with $k=1, \ldots, N$, proceeds as follows. The measured raw image $I_{i, j}$ is processed. The processing steps include (1) the removal of randomly occurring black and white pixels (usually referred to as "salt and pepper" noise) by median filtering, (2) the subtraction of a background image (i.e. an image without photoemitted beam), and (3) the application of a thresholding (i.e. the elements in the image array below a user-defined value are zeroed). Finally a region of interest can be selected. The thereby obtained matrix, $I_{i, j}$, with its values normalized such that $\max \left[I_{i, j}\right]=1$, is then used as the discretized density function (the maximum size of the array is $640 \times 480$ corresponding to the CCDs pixel array). In the following Monte Carlo algorithm: (1) A triplet of real random numbers $(x, y, J)$ uniformly distributed in the respective intervals $x \in[0,639], y \in[0,479]$, and $J \in[0,1]$ is generated. We used the Sobol sequence [36] to generate the quasirandom sequence of numbers to achieve more uniformly spaced samples of triplets. (2) If the condition $I_{\text {round }(x) \text {,round }(y)}>J$, then the triplet is considered valid and a macroparticle with transverse coordinates $(x, y)$ is added to the initial distribution. (3) If the aforementioned condition is not fulfilled, then the $(x, y, J)$ triplet is rejected and step (1) is repeated. (4) Steps (1), (2), and (3) are repeated until $N$ macroparticles are generated. Finally, the coordi-
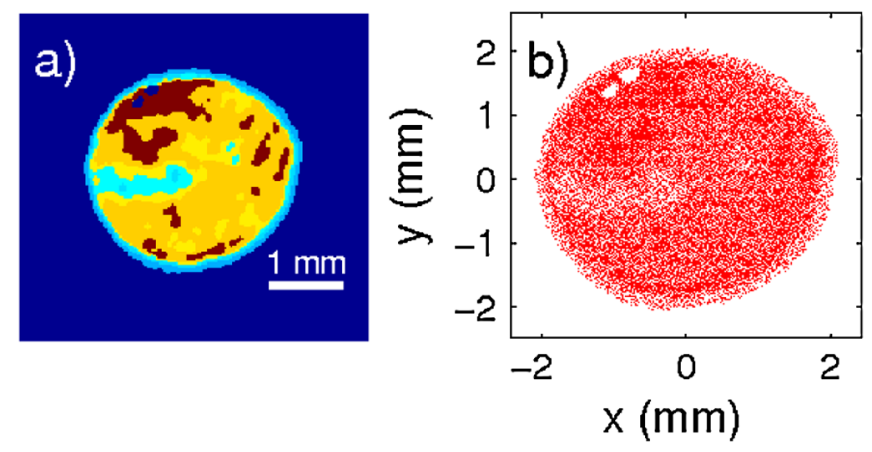

FIG. 14. (Color) Example of measured transverse laser intensity distribution (a) and corresponding generated macroparticle distribution (b). Note that only 20000 macroparticles are displayed for clarity. nates of the macroparticles are scaled per the calibration of the CCD camera measurement. An example of the measured image along with the associated macroparticle transverse distributions generated with the above algorithm is presented in Fig. 14.

Similarly, the measured and processed 1D temporal distribution, $S_{q}$, can be used to generated the longitudinal coordinates of the macroparticles. However, because of the Gaussian temporal shape of the laser pulse we used the measured rms duration $\sigma_{t}$ to directly generate time coordinates that have a normal distribution using the BoxMuller [37] transformation: two independent real variables $u$ and $v$ randomly distributed in $[0,1]$ are combined to generate the coordinate $t_{i}$ via

$$
t_{i}=\sigma_{t} \sqrt{-2 \log u_{i}} \cos \left(2 \pi v_{i}\right)
$$

[1] See, for instance, J. Arthur et al., LCLS Conceptual Design Report, Report No. SLAC-R-593/UC-414, 2002; TESLA Technical Design Report Imprint No. DESY 2001-011 or ECFA 2001-209, edited by R. Brinkmann et al., 2001.

[2] See the web site http://www.linearcollider.org.

[3] W. Gai, P. Schoessow, B. Cole, R. Konecny, J. Norem, J. Rosenzweig, and J. Simpson, Phys. Rev. Lett. 61, 2756 (1988).

[4] R. L. Gluckstern, Phys. Rev. Lett. 73, 1247 (1994).

[5] S. Bernal, R. A. Kishek, and M. Reiser, in Proceedings of the 18th Particle Accelerator Conference, New York, 1999 (IEEE, New York, 1999), p. 1749.

[6] J.S. Fraser, R. L. Scheffield, E. R. Gray, and G. W. Rodenz, in Proceedings of the 1985 IEEE Particle Accelerator Conference (IEEE, Vancouver, BC, 1985), p. 1791.

[7] F. Zhou, I. Ben-Zvi, M. Babzien, X. Y. Chang, A. Doyuran, R. Malone, X.J. Wang, and V. Yakimenko, Phys. Rev. ST Accel. Beams 5, 094203 (2002).

[8] C. Lejeune and J. Aubert, Adv. Electron. Electron Phys. Suppl. A 13, 159 (1980).

[9] F. R. Sacherer, IEEE Trans. Nucl. Sci. 18, 1105 (1971).

[10] M. Reiser et al., Phys. Rev. Lett. 61, 2933 (1988).

[11] I. Haber et al., Phys. Rev. A 44, 5194 (1991).

[12] D. Stratakis, R. A. Kishek, I. Haber, S. Bernal, M. Reiser, and P. G. O'Shea, Phys. Rev. ST Accel. Beams 12, 064201 (2009).

[13] V. Shiltsev, Nucl. Instrum. Methods Phys. Res., Sect. A 374, 137 (1996).

[14] V. Shiltsev et al., Phys. Rev. ST Accel. Beams 11, 103501 (2008).

[15] C. M. Celata et al., Phys. Plasmas 10, 2064 (2003).

[16] T. Kondoh, et al., in Proceedings of the 11th European Particle Accelerator Conference, Genoa, 2008 (EPS-AG, Genoa, Italy, 2008), p. 1809.

[17] Information on the Argonne Wakefield Facility is available at the web site http://gate.hep.anl.gov/awa.

[18] The Ce:YAG screens were procured from Crytur Ltd.; see the web site http://www.crytur.cz/. 
[19] The ICT is manufactured by Bergoz Instrumentation; see the web site http://www.bergoz.com/.

[20] J. G. Power, H. Wang, M. E. Conde, W. Gai, R. Konecny, W. Liu, and Z. Yusof, Nucl. Instrum. Methods Phys. Res., Sect. A 546, 345 (2005).

[21] N. Brown and M. Reiser, Phys. Rev. Lett. 71, 2911 (1993); see also Ref. [24], Sec. 4.4.5.

[22] J. H. Billen and L. M. Young, in Proceedings of the 1993 Particle Accelerator Conference, Washington, DC (IEEE, Piscataway, NJ, 1993), p. 790.

[23] I. N. Meshkov, Transport of the Charged Particles (Budker INP, Novosibirsk, 1989) (in Russian).

[24] M. Reiser, The Theory and Design of Charged Particle Beams, Wiley Series in Beam Physics and Accelerator Technology (Wiley, New York, 1994); see Eq. 3.59, p. 74.

[25] J. Qiang, S. Lidia, R. D. Ryne, and C. Limborg-Deprey, Phys. Rev. ST Accel. Beams 9, 044204 (2006).

[26] H. Shang and M. Borland, in Proceedings of the 21st Particle Accelerator Conference, Knoxville, 2005 (IEEE, Piscataway, NJ, 2005), p. 4230.

[27] M. Rihaoui, W. Gai, P. Piot, J. Power, and Z. Yusof, Proceedings of the 2009 Particle Accelerator Con- ference, Vancouver, BC (to be published).

[28] B. E. Carlsten, Nucl. Instrum. Methods Phys. Res., Sect. A 285, 313 (1989).

[29] L. Serafini and J. B. Rosenzweig, Phys. Rev. E 55, 7565 (1997).

[30] C.-x. Wang, Phys. Rev. E 74, 046502 (2006).

[31] G. Geloni, E. Saldin, E. Schneidmiller, and M. Yurkov, Nucl. Instrum. Methods Phys. Res., Sect. A 554, 20 (2005).

[32] J. Wu, Z. Huang, and P. Emma, Phys. Rev. ST Accel. Beams 11, 040701 (2008).

[33] J. Qiang et al., Nucl. Instrum. Methods Phys. Res., Sect. A 457, 1 (2001).

[34] See, for instance, R. W. Howckney and J. W. Eastwood, Computer Simulation Using Particles (Adam Higler, Bristol and New York, 1989).

[35] B. E. Carlsten, Part. Accel. 49, 34 (1995).

[36] W. H. Press, Brian P. Flannery, S. A. Teukolsky, and W. T. Vetterling, Numerical Recipes in C (Cambridge University Press, Cambridge, UK, 1992), 2nd ed.

[37] G. E. P. Box and M.E. Muller, Ann. Math. Stat. 29, 610 (1958). 